\title{
An Analytical and Numerical Investigation of the Optical Vortex Solitons: A Computational Study
}

\author{
Seyedali Vedad (Corresponding author) \\ Institute for Advanced Studies \\ Tehran 14456-63543, Iran
}

Tel: 98-912-102-3131_Email: Seyedali.Vedad@yahoo.com

\author{
Alireza Heidari \\ Institute for Advanced Studies \\ Tehran 14456-63543, Iran
}

Tel: 98-912-102-3131Ｅmail: Prof.Alireza.Heidari@InstituteforAdvancedStudies.us

\author{
Received: November 28, 2011 Accepted: December 11, 2011 Published: February 1, 2012 \\ doi:10.5539/apr.v4n1p78 \\ URL: http://dx.doi.org/10.5539/apr.v4n1p78
}

\begin{abstract}
In this paper, we investigate the possibility of forming spatiotemporal vortex solitons in the dispersive inhomogeneous non-linear optical fibers using a graded-index Kerr medium as an example. Also, we use a variation approach to solve the multidimensional inhomogeneous, the non-linear Schrödinger equation (NLSE) and show that spatiotemporal vortex solitons can be stabilized under certain conditions.
\end{abstract}

Keywords: Singularity, Soliton, Light bullet, Vortex

\section{Introduction}

Over the past decade, the optical temporal-spatial vortex solitons called "Vortex Optical Bullets" have attracted a great deal of interest. They are multidimensional pulses whose shape will be remained unchanged during the time and in a spatial dimension due to the balance among the group-velocity dispersion, diffraction, and phase modulation; furthermore, they possess phase unity (Russell, 1844; Schiesser, 1994; Wadati, 2001; Mollenauer, et al., 1980; Desyatnikov, et al., 2005; Kosterlitz \& Thouless, 1973).

Studying the optical vortexes is of importance in terms of pure and applied physics. It is expected that the unique nature of vortex fields result in applications in various fields such as storing the optical data and processing them. Because the vortex solitons can turn during the propagation in the non-linear medium, this motion may be used for making the optical rotational switching based on the vortex solitons' concept (Carlsson, 2000). Owing to the temporal-spatial solitons' extremely fast all-optical switching ability in a mass medium, they are expected to appear as a new fundamental physical creature in the photonic. Among other applications, the optical bullets are of great significance in the all-optical digital logic (Kivshar \& Agrawal, 2003; Young, 1802; Whewell, 1833; Winfree, 1995; Soskin \& Vasnetsov, 2001; Nye \& Berry, 1974; Pismen, 1999).

The optical vortexes can be applied for trapping tiny particles. Also, another special application of optical temporal-spatial vortex solitons includes conducting the waves as the waveguide. Take the step-index waveguides for instance, due to their variable refraction index in different points of the medium resulted from its structure, they lead to light conduction and formation of waveguide. In the presence of optical vortex, its propagation in the medium can induce the inclined refraction index in the medium, and consequently, as it is conducted in the medium, it conducts other waves and creates a waveguide bringing about producing all-optical circuits (Carlsson, 2000).

The optical temporal-spatial vortex solitons in the third-order non-linear and dispersive media have been previously studied. Here, we assume that the studied medium is heterogeneous besides having those properties. Therefore, we want to investigate the possibility of forming the optical temporal-spatial vortex solitons in the 
dispersive heterogeneous optical fibers with the third-order non-linearity (Kerr medium). To solve the multidimensional non-linear Schrödinger equation, we utilize a variational method, and indicate that the optical temporal-spatial vortex solitons can be stable under certain conditions (Mihalache, et al., 2006; 2002; Novikov, 1984; Hasegawa \& Tappert, 1973; Iannone, et al., 1998; Agrawal, 1989; Bergé, 1998; Jackiw \& So-Young, 1990; Nore, et al., 1993; Velchev, 1997; Chen \& Atai, 1992; Tikhonenko, et al., 1998; Kivshar, et al., 1998; Królikowski, et al., 1998; Kivshar \& Yang, 1994; Rubinstein \& Pismen, 1994; Luther-Davies, et al., 1994; Staliunas, 1994; Butylkin, et al., 1989; Kuznetsov, et al., 1986; Kuznetsov \& Turitsyn, 1988; Kivshar \& Yang, 1994; Mamaev,et al., 1996; Lugomer, et al., 1998; Law \& Swartzlander, 1994; Tilkhonenko, et al., 1996; Swartzlander, 1992; Luther-Davies, et al., 1997;Mamaev, et al., 1996; Kivshar \& Luther-Davies, 1998; Neshev, et al., 2004; Kivshar \& Peyrard, 1992; Berry, 1984; Desyatnikov \& Kivshar, 2001).

\section{The Non-linear Schrödinger Equation (NLSE)}

In order to study the vortex optical bullets in the fiber non-linear medium, we assume that the medium possesses Kerr non-linearity, and in contrast with the studied cases, we consider the medium to be heterogeneously. Consequently, the refraction index of such medium can be given by:

$$
n(r, \omega)=n_{0}(\omega)+n_{1}\left(x^{2}+y^{2}\right)+n_{2}|E|^{2}
$$

where the homogenous part $n_{0}(\omega)$ results from the color dispersion, $n_{2}$ results from the non-linear response of the self-focusing or self-defocusing medium, and $n_{1}$ is the changes in the refraction index in the transverse dimensions of $x$ and $y$. We assume that the light propagates along the axis $z$. When $n_{1}>0$, the medium is conductive, and when $n_{1}<0$, the medium is anti-conductive.

We begin by analyzing Eq. (1) using the Maxwell equations. The oscillating electric field in the frequency $\omega_{0}$ can be considered as:

$$
E(r, t)=\frac{1}{2} \hat{e} A^{\prime}(r, t) \exp \left[i\left(\beta_{0} z-\omega_{0} t\right)\right]+c . c .
$$

where $\hat{e}$ is the unit vector, $\beta_{0}=n_{0}\left(\omega_{0}\right) k_{0}$, the wave number $k_{0}=\omega / c$, and $A^{\prime}(r, t)$ is the field envelope. Applying the slowly varying envelope approximations, we will attain the equation governing the envelope. We begin with the wave equation:

$$
\nabla^{2} \vec{E}-\frac{n^{2}}{c^{2}} \frac{\partial^{2} \vec{E}}{\partial t^{2}}=0
$$

From Eq. (1), we have:

$$
\begin{gathered}
n^{2}(r, \omega)=\left[n_{0}(\omega)+n_{1}\left(x^{2}+y^{2}\right)+n_{2}|E|^{2}\right]^{2} \\
=n_{0}{ }^{2}\left(\omega_{0}\right)+n_{1}{ }^{2}\left(x^{2}+y^{2}\right)^{2}+n_{2}{ }^{2}|E|^{4}+2 n_{0}\left(\omega_{0}\right) n_{1}\left(x^{2}+y^{2}\right)+2 n_{0}\left(\omega_{0}\right) n_{2}|E|^{2}+2 n_{1}\left(x^{2}+y^{2}\right) n_{2}|E|^{2}
\end{gathered}
$$

In the above-mentioned terms, we can ignore the orders higher than $n_{1}$ and $n_{2}$. The wave equation's derivations are calculated as follows:

$$
\begin{gathered}
\frac{\partial^{2} E}{\partial x^{2}}=\frac{1}{2} \hat{e}\left\{\frac{\partial^{2} A}{\partial x^{2}} \exp i\left(\beta_{0} z-\omega_{0} t\right)+C . C\right\} \\
\frac{\partial^{2} E}{\partial y^{2}}=\frac{1}{2} \hat{e}\left\{\frac{\partial^{2} A}{\partial y^{2}} \exp i\left(\beta_{0} z-\omega_{0} t\right)+C . C\right\} \\
\frac{\partial^{2} E}{\partial z^{2}}=\frac{1}{2} \hat{e}\left\{2 i \beta_{0} \frac{\partial A}{\partial z} \exp i\left(\beta_{0} z-\omega_{0} t\right)-\beta_{0}^{2} A \exp i\left(\beta_{0} z-\omega_{0} t\right)+C . C\right\}
\end{gathered}
$$




$$
\frac{\partial^{2} E}{\partial t^{2}}=\frac{1}{2} \hat{e}\left\{\frac{\partial^{2} A}{\partial t^{2}} \exp i\left(\beta_{0} z-\omega_{0} t\right)-2 i \omega_{0} \frac{\partial A}{\partial t} \exp i\left(\beta_{0} z-\omega_{0} t\right)-\omega_{0}^{2} A \exp i\left(\beta_{0} z-\omega_{0} t\right)+C . C\right\}
$$

Inputting Eqs. (4) and (5) to Eq. (3), we have:

$$
\begin{aligned}
& \frac{1}{2 \beta_{0}} \nabla_{\perp}^{2} A^{\prime}+i \frac{\partial A^{\prime}}{\partial z}-\frac{1}{2 \beta_{0}} \frac{n_{0}^{2}}{c^{2}} \frac{\partial^{2} A^{\prime}}{\partial t^{2}}-\frac{n_{0} n_{1}\left(x^{2}+y^{2}\right)}{\beta_{0} c^{2}} \frac{\partial^{2} A^{\prime}}{\partial t^{2}}-\frac{n_{0} n_{2}|E|^{2}}{\beta_{0} c^{2}} \frac{\partial^{2} A^{\prime}}{\partial t^{2}}+i \omega_{0} \frac{n_{0}{ }^{2}}{\beta_{0} c^{2}} \frac{\partial A^{\prime}}{\partial t} \\
& +i \omega_{0} \frac{2 n_{0} n_{1}\left(x^{2}+y^{2}\right)}{\beta_{0} c^{2}} \frac{\partial A^{\prime}}{\partial t}+i \omega_{0} \frac{2 n_{0} n_{2}|E|^{2}}{\beta_{0} c^{2}} \frac{\partial A^{\prime}}{\partial t}+A^{\prime} n_{1}\left(x^{2}+y^{2}\right) k_{0}+k_{0} n_{2}\left|A^{\prime}\right| A^{\prime}=0
\end{aligned}
$$

where $\beta_{0}=n_{0}\left(\omega_{0}\right) k_{0}, k_{0}=\omega / c$, and $\nabla_{\perp}^{2}$ is the transverse Laplacian operator defined as $\nabla_{\perp}^{2}=\frac{\partial^{2}}{\partial x^{2}}+\frac{\partial^{2}}{\partial y^{2}}$.

From Eq. (2), we can pull out the terms including $i \frac{\partial A^{\prime}}{\partial t}$, and then we have:

$$
\left\{\omega_{0} \frac{n_{0}^{2}}{\beta_{0} c^{2}}+\omega_{0} \frac{2 n_{0} n_{1}\left(x^{2}+y^{2}\right)}{\beta_{0} c^{2}}+\omega_{0} \frac{2 n_{0} n_{2}|E|^{2}}{\beta_{0} c^{2}}\right\} i \frac{\partial A^{\prime}}{\partial t}=\left\{\frac{n_{0}}{c}+\frac{2 n_{1}\left(x^{2}+y^{2}\right)}{c}+\frac{2 n_{2}|E|^{2}}{c}\right\} i \frac{\partial A^{\prime}}{\partial t}
$$

We know that:

$$
\beta=\frac{n(\omega) \omega}{c} ; \quad \beta_{1}=\left.\frac{d \beta}{d \omega}\right|_{\omega=\omega_{0}} \quad ; \quad \beta_{2}=\left.\frac{d^{2} \beta}{d \omega^{2}}\right|_{\omega=\omega_{0}} \quad ; \quad \beta_{1}=\frac{n(\omega)}{c}+\left.\frac{d n}{d \omega} \frac{\omega}{c}\right|_{\omega=\omega_{0}}
$$

Now, using the Caushi equation, we have:

$$
\frac{d n}{d \omega}=A^{\prime} \omega
$$

It can be written:

$$
\beta_{1}=\frac{n(\omega)}{c}+\frac{A^{\prime} \omega^{2}}{c}=\frac{n_{0}(\omega)+n_{1}\left(x^{2}+y^{2}\right)+n_{2}|E|^{2}+A^{\prime} \omega^{2}}{c}
$$

Also, we assume the following equation is established:

$$
A^{\prime} \omega^{2}=n_{1}\left(x^{2}+y^{2}\right)+n_{2}|E|^{2}
$$

This assumption is based on this principle that to form the temporal-spatial solitons, in Eq. (10), the spatial and temporal parts of $n_{2}|E|^{2}$ are neutralized with $n_{1}\left(x^{2}+y^{2}\right)$ and $A^{\prime} \omega^{2}$, respectively. Therefore, we have:

$$
\beta_{1}=\frac{n_{0}(\omega)}{c}+\frac{2 n_{1}\left(x^{2}+y^{2}\right)}{c}+\frac{2 n_{2}|E|^{2}}{c}
$$

where this is the coefficient of $i \frac{\partial A^{\prime}}{\partial t}$ mentioned in Eq. (6).

Accordingly, we pull out the terms including $\frac{\partial^{2} A^{\prime}}{\partial t^{2}}$ in Eq. (6), and write:

$$
\left\{\frac{1}{2 \beta_{0}} \frac{n_{0}^{2}}{c^{2}}+\frac{n_{0} n_{1}\left(x^{2}+y^{2}\right)}{\beta_{0} c^{2}}+\frac{n_{0} n_{2}|E|^{2}}{\beta_{0} c^{2}}\right\} \frac{\partial^{2} A^{\prime}}{\partial t^{2}}=\frac{1}{2 \omega_{0} c}\left\{n_{0}+2 n_{1}\left(x^{2}+y^{2}\right)+2 n_{2}|E|^{2}\right\} \frac{\partial^{2} A^{\prime}}{\partial t^{2}}
$$

On the other hand: 


$$
\beta_{2}=\frac{2}{c} \frac{d n}{d \omega}+\left.\frac{\omega}{c} \frac{d^{2} n}{d \omega^{2}}\right|_{\omega=\omega_{0}}
$$

Employing the Caushi equation mentioned in Eq. (9), the above equation is rewritten as the form below:

$$
\beta_{2}=\frac{2}{c} \frac{d n}{d \omega}+\left.\frac{\omega}{c} \frac{d^{2} n}{d \omega^{2}}\right|_{\omega=\omega_{0}}=\frac{2}{c} \frac{A^{\prime} \omega^{2}}{\omega}+A^{\prime} \frac{\omega^{2}}{c \omega}
$$

Using Eq. (11), we obtain:

$$
\beta_{2}=\frac{2}{c} \frac{n_{1}\left(x^{2}+y^{2}\right)+n_{2}|E|^{2}}{\omega}+\frac{A^{\prime} \omega^{2}}{c \omega}
$$

The next assumption is as follows:

$$
n_{0}(\omega)=A^{\prime} \omega^{2}
$$

Actually, this assumption is correct owing to the fact that the dispersion equation for $n$ is the same as the dispersion equation for $n_{0}$ because $n_{0}$ is the only function of $\omega$, and any $\omega$ functionality is not appeared in $n_{1}$ and $n_{2}$.

Generally, the Caushi equation is written as the following form:

$$
n=A+\frac{B}{\lambda^{2}}
$$

from which Eq. (9) derives, and Eq. (11) is the same as the Caushi equation without the constant $A$. Consequently, we have:

$$
\beta_{2}=\frac{1}{\omega c}\left\{n_{0}(\omega)+2 n_{1}\left(x^{2}+y^{2}\right)+2 n_{2}|E|^{2}\right\}
$$

It means that the coefficient of $\frac{\partial^{2} A^{\prime}}{\partial t^{2}}$ in Eq. (6) is $\frac{\beta_{2}}{2}$.

Through inserting $\beta_{1}$ and $\beta_{2}$ into Eq. (2), we have:

$$
i\left(\frac{\partial A^{\prime}}{\partial z}+\beta_{1} \frac{\partial A^{\prime}}{\partial t}\right)-\frac{\beta_{2}}{2} \frac{\partial^{2} A^{\prime}}{\partial t^{2}}+\frac{1}{2 \beta_{0}} \nabla_{\perp}^{2} A^{\prime}+k_{0} n_{1}\left(x^{2}+y^{2}\right) A^{\prime}+k_{0} n_{2}\left|A^{\prime}\right|^{2} A^{\prime}=0
$$

The above equation, which will be used in the vortex solitons' equation, is the same as the equation governing the electric field envelope.

\section{The Mathematical forMulating of Vortex Optical Bullets}

To find the solutions to Eq. (20), we initially normalize this equation. We need to consider some quantities to normalize. We define the transverse (length) scale and transverse-coordinates scale as $h_{0}=\left(2 k_{0}\left|n_{1}\right| \beta_{0}\right)^{-1 / 4}$ and $(X, Y)=(x, y) / h_{0}$, respectively. Similarly, the longitudinal (length) scale and longitudinal-coordinates scales are respectively defined as $L_{d}=\beta_{0} h_{0}^{2}$ (the diffraction length) and $Z=z / L_{d}$. Also, the time is scaled as $\tau=\left(t-\beta_{1} z\right) / T_{0}$ where $T_{0}=\sqrt{\left|\beta_{2}\right|} L_{d}$.

Regarding the introduced quantities, the derivatives with the following equations are related to the new coordinates:

$$
\frac{\partial^{2}}{\partial x^{2}}=\left(\frac{1}{\omega_{0}}\right)^{2} \frac{\partial^{2}}{\partial X^{2}} ; \quad \frac{\partial^{2}}{\partial y^{2}}=\left(\frac{1}{\omega_{0}}\right)^{2} \frac{\partial^{2}}{\partial Y^{2}}
$$

and also: 


$$
\frac{\partial}{\partial z}=\frac{1}{L_{d}} \frac{\partial}{\partial Z}-\frac{\beta_{1}}{T_{0}} \frac{\partial}{\partial \tau} ; \quad \frac{\partial^{2}}{\partial t^{2}}=\left(\frac{1}{T_{0}}\right)^{2} \frac{\partial^{2}}{\partial \tau^{2}}
$$

According to the above equations, Eq. (20) is transformed into the normalized form below:

$$
i \frac{\partial U}{\partial Z}+\frac{1}{2}\left(\nabla_{\perp}^{2} U\right)+\frac{\delta}{2} \frac{\partial^{2} U}{\partial \tau^{2}}-\frac{s}{2} r^{2} U+v|U|^{2} U=0
$$

where $U(X, Y, Z, \tau)=\sqrt{k_{0} n_{2} L_{d}} A^{\prime}(x, y, z, t)$, and the parameter $r$ is a coordinate of the polar coordinates which being related to the transverse coordinates through $r^{2}=X^{2}+Y^{2}$. Moreover, the parameters $\delta= \pm 1$, $s= \pm 1$, and $v= \pm 1$ respectively denote $\beta_{2}, n_{1}$, and $n_{2}$ which imply the medium with normal or abnormal group-velocity dispersion, conductive or anti-conductive medium, and self-focusing or self-defocusing medium, respectively. This equation is similar to the multidimensional standard non-linear Schrödinger equation (NLSE) and the main difference relates to the heterogeneity of non-linear medium.

It can be indicated that the following Lagrangian can be attributed to the above non-linear Schrödinger equation because the above equation is considered as the motion equation and the same answers can be achieved by both equations:

$$
l=-\operatorname{Im}\left(U^{*} \frac{\partial U}{\partial Z}\right)-(H)
$$

where $H$ is the system's Hamiltonian. We have the Lagrangian opened form below:

$$
l=-\operatorname{Im}\left(U^{*} \frac{\partial U}{\partial Z}\right)+\left(\frac{1}{2}|\nabla U|^{2}+\frac{\delta}{2}\left|\frac{\partial U}{\partial \tau}\right|^{2}+\frac{s}{2} r^{2}|U|^{2}-\frac{v}{2}|U|^{4}\right)
$$

We know that the Euler's theorem is written as follows:

$$
\frac{d}{d q_{k}}=\left(\frac{\partial l}{\partial U / \partial q_{k}}\right)-\frac{\partial l}{\partial U^{*}}=0 ; \quad q_{k}=X, Y, Z, \tau
$$

Therefore, utilizing Eq. (25) and applying the Euler's theorem, the following equations can be obtained:

$$
\begin{gathered}
\frac{d}{d X}\left(\frac{\partial l}{\partial U / \partial X}\right)=\frac{1}{2} \frac{\partial^{2} U}{\partial X^{2}} \\
\frac{d}{d Y}\left(\frac{\partial l}{\partial U / \partial Y}\right)=\frac{1}{2} \frac{\partial^{2} U}{\partial Y^{2}} \\
\frac{d}{d Z}\left(\frac{\partial l}{\partial U / \partial Z}\right)=0 \\
\frac{d}{d \tau}\left(\frac{\partial l}{\partial U / \partial \tau}\right)=\frac{1}{2} \frac{\partial^{2} U}{\partial \tau^{2}}
\end{gathered}
$$

Through inputting the above-mentioned equations to the Euler's theorem, we reach to Eq. (23) that this case indicates the Lagrange equation's accuracy for this system, and consequently, the Lagrangian's correctness becomes validated.

Selecting the trial solution is the determinant factor in the variation method's success. In Eq. (23), it can be observed that, in the temporal dimension, the combination of group-velocity dispersion and self-defocusing can lead to the sech light soliton. In addition, in the transverse dimensions of waveguide, the gradual index is turned to the Hermite-Gaussian modes such that the basic mode is in the Gaussian form. In the absence of such trapping, the temporal and spatial dimensions must behave symmetrically; however, because the transverse 
trapping is strong, we consider the trial function as the product of a chirped sech pulse and a chirped Gaussian beam. Furthermore, we add the singularity with a power such that:

$$
U(X, Y, Z, \tau)=A \operatorname{sech}\left(\frac{\tau}{w}\right) r^{m} \exp \left(-\frac{r^{2}}{2 a^{2}}+i \Phi\right)
$$

where $\Phi=m \theta+\alpha r^{2}+\beta \tau^{2}+\varphi$ is the phase function. $\alpha(Z)$ and $\beta(Z)$ are the wavefront curvatures (chirps), $\varphi(Z)$ is the free phase, $\theta=\tan ^{-1}(Y / X)$ is a coordinate of the cylindrical coordinates, and $m$ is an integer that indicates the phase wrap around the intensity loop, and is called the vortex topological charge. In addition to the radial modes, such a number determines the higher-order directional steady states. We normalize $U$ such that $\int|U|^{2} d X d Y d \tau=\varepsilon$. By this definition and using the above-mentioned integrals, the pulse energy constant is found that equals $\varepsilon=2 \pi m ! A^{2} a^{2 m+2} w$. Therefore, we replace the constant $A$ in Eq. (31) with its equivalent, $A=\sqrt{\varepsilon / 2 \pi m ! a^{2 m+2} w}$.

In accordance with the standard method, the effective Lagrangian is calculated as follows:

$$
L=\langle l\rangle=\int l\left(u, u^{*}\right) d X d Y d \tau
$$

Through inserting the Lagrangian of Eq. (25) into Eq. (32) and integrating, we obtain some integrals such as the temporal integrals:

$$
\begin{gathered}
\int \operatorname{sech}^{2}(\eta \tau) d \tau=\frac{2}{\eta} \\
\int \operatorname{sech}^{4}(\eta \tau) d \tau=\frac{4}{3 \eta} \\
\int \operatorname{sech}^{2}(\eta \tau) \tanh ^{2}(\eta \tau) d \tau=\frac{2}{3 \eta} \\
\int \tau^{2} \operatorname{sech}^{2}(\eta \tau) d \tau=\frac{\pi^{2}}{6 \eta^{3}}
\end{gathered}
$$

and also, the spatial integrals which have a solution proportional to the Gama function:

$$
\begin{gathered}
\int_{0}^{\infty} \exp (-u) u^{n} d u=2 \int_{0}^{\infty} \exp \left(-u^{2}\right) u^{2 n+1} d u=n !=\Gamma(n+1) \\
\int \exp \left[-\frac{x^{2}+y^{2}}{a^{2}}\right]\left(x^{2}+y^{2}\right)^{m} d x d y=\int \exp \left[-\frac{\rho^{2}}{a^{2}}\right]\left(\rho^{2}\right)^{m} \rho d \rho d \theta=\pi a^{2(m+1)} \Gamma(m+1) \\
\int \exp \left[-\frac{x^{2}+y^{2}}{a^{2}}\right]\left(x^{2}+y^{2}\right)^{m-1} d x d y=\pi a^{2 m} \Gamma(m) \\
\int \exp \left[-\frac{x^{2}+y^{2}}{a^{2}}\right]\left(x^{2}+y^{2}\right)^{m+1} d x d y=\pi a^{2(m+2)} \Gamma(m+2) \\
\int \exp \left[-\frac{2\left(x^{2}+y^{2}\right)}{a^{2}}\right]\left(x^{2}+y^{2}\right)^{2 m} d x d y=\pi \frac{a^{2(2 m+1)}}{2^{2 m+1}} \Gamma(2 m+1)
\end{gathered}
$$

Consequently, using the above-mentioned equations, the effective Lagrangian is obtained as follows:

$$
L=\varepsilon\left\{(m+1) a^{2} \frac{d \alpha}{d Z}+\frac{w^{2} \pi^{2}}{12} \frac{d \beta}{d Z}+\frac{d \varphi}{d Z}\right.
$$




$$
\left.+\frac{1}{2}\left[4 \alpha^{2} a^{2}(m+1)+\frac{(m+1)}{a^{2}}+\frac{\delta}{3 w^{2}}+\frac{\delta \beta^{2} w^{2} \pi^{2}}{3}+s(m+1) a^{2}\right]-\frac{1}{2^{2 m+1}} \frac{v \varepsilon}{6 w a^{2} \pi} \frac{(2 m) !}{(m !)^{2}}\right\}
$$

Then, by the means of the Lagrange-Euler equations, the following equations are achieved:

$$
\begin{gathered}
\frac{d w}{d Z}=2 \beta \delta w \\
\frac{d a}{d Z}=2 a \alpha \\
\frac{d \beta}{d Z}=\frac{2 \delta}{\pi^{2} w^{4}}-2 \delta \beta^{2}-\frac{2 v \tilde{\varepsilon}}{\pi^{2} w^{3} a^{2}} \\
\frac{d \alpha}{d Z}=-2 \alpha^{2}-\frac{s}{2}+\frac{1}{2 a^{4}}-\frac{v \tilde{\varepsilon}}{3(m+1) w a^{4}}
\end{gathered}
$$

where $\tilde{\varepsilon}=\varepsilon(2 m) ! / 2^{2 m+2} \pi(m !)^{2}$

Now, using the above-mentioned results, it can be indicated that the paired equations governing on the spatial width $a$ and pulse durability $w$ include:

$$
\begin{gathered}
\frac{d^{2} a}{d Z^{2}}=-s a+\frac{1}{a^{3}}-\frac{2}{3} \frac{v \tilde{\varepsilon}}{(m+1) a^{3} w} \equiv I(a, w) \\
\frac{d^{2} w}{d Z^{2}}=\frac{4}{\pi^{2}}\left(\frac{1}{w^{3}}-\frac{v \delta \tilde{\varepsilon}}{w^{2} a^{2}}\right) \equiv J(a, w)
\end{gathered}
$$

The first term at the right side of Eqs. (47) and (48) are respectively related to the diffraction and dispersion, and the last terms being proportional to the pulse energy indicate the effect of non-linearity.

\section{Results and Discussion}

The solitonic answers $w_{0}$ and $a_{0}$ can be obtained at the steady limit of Eqs. (47) and (48), when their left side tends to zero. For $s=0$, the replaced transverse answers, $a_{0}<\infty$, solely exist for $v=+1$ (self-focusing media). While for $s=1$, ignoring the sign of $v$, the field becomes trapped in the waveguide. Particularly for $\varepsilon=0$, the linear conducted mode with $a_{0}=1$ is appeared as the continuous answer to the work with $w_{0}=\infty$. In contrast, the solitonic pulses with the final width, $w_{0}=\frac{a_{0}^{2}}{\delta v \tilde{\varepsilon}}<\infty$, exist only when $\delta v=+1$. In the steady state, the spatial width is given by the positive roots of the equation below:

$$
s a^{\prime 3}-a^{\prime}+\gamma=0
$$

where $a^{\prime}=a_{0}^{2}$ and $\gamma=2 \delta \tilde{\varepsilon}^{2} / 3(m+1)$. For $s=0$, the rotating optical bullets are only the steady answers for $\delta=v=+1$, and $a_{0}^{2}=2 \tilde{\varepsilon}^{2} / 3(m+1)$ [50-52]. For $s=1$, the above third-order polynominal has no positive answer in $\gamma>2 / 3 \sqrt{3}$, and the first answer, $a_{0}^{2}=1 / \sqrt{3}$, appears in $\gamma=2 / 3 \sqrt{3}$. More decreasing the pulse energy, $0<\gamma<2 / 3 \sqrt{3}$, we will have two answers and the vortex soliton will become double steady. Then through passing the linear conducted mode $\gamma=0$ and reversing the sign of non-linearity, $\gamma<0$ and $\delta=-1$, a singular answer similar to the reference (Raghavan \& Agrawal, 2000) is obtained.

Here, we consider the non-linear medium to be the self-defocusing medium, and apply it to the illustration of diagrams by considering $v=-1$. As mentioned, the solitonic pulses with the final width, $w_{0}=a_{0}^{2} / \delta v \tilde{\varepsilon}$, exist only when $\delta v=+1$, because the width is always, $w_{0}<\infty$, a positive value. Therefore, this condition causes $\delta=-1$, 
and also, this suggests that the medium is a medium with normal group-velocity dissemination. Moreover, we consider the non-linear medium to be a conductive medium which it requires applying the condition $s=+1$.

In Fig. 1, the vortex solitons for different topological charges are illustrated. In the part (a), it is observed that, for the small $r$ (representing the vortex core), the intensity is zero. This case is clearly shown in the intensity distribution graph, Fig. 1b, for one of the topological charges. This intensity graph for each topological charge is as the following form. It can be observed that, for the higher order of $m$, the vortex soliton resembles loops around the center, the same as donut.

Next, we study the changes in the spatial width and the durability of optical temporal-spatial vortex soliton in the steady state and according to the pulse energy. Fig. (2) indicates that the rate of changes in the pulse spatial width according to the energy decline by increasing the order of topological charge, and inclines towards the lim $a_{0} \rightarrow 1$ which is the same as the limit of linear conducted mode or continuous mode of work.

Fig. 3 indicates the changes in the pulse durability according to the pulse energy for different topological charges. As it is evident, the energy threshold corresponding to the continuous state of work, $w_{0} \rightarrow \infty$, rises with increasing the topological charge.

To investigate the stability of temporal-spatial solitons, it is necessary for Eqs. (47) and (48) to become linear around the steady point. For this purpose, we consider the answers as $a(Z)=a_{0}+p(Z)$ and $w(Z)=w_{0}+q(Z)$ such that $p(Z) \square a_{0}$ and $q(Z) \square w_{0}$ are considered as the disturbance terms.

For the above small disturbances, Eqs. (47) and (48) can be indicated as the following matrix:

$$
\frac{d^{2}}{d Z^{2}}\left(\begin{array}{l}
p \\
q
\end{array}\right)=\left(\begin{array}{ll}
I_{a} & I_{w} \\
J_{a} & J_{w}
\end{array}\right)\left(\begin{array}{l}
p \\
q
\end{array}\right)
$$

where the quantities of $J_{w}, J_{a}, I_{w}$, and $I_{a}$ are the partial derivatives of $J, I$ with respect to $a, w$, spatial width, and pulse durability in the steady values. The eigenvalues of above-mentioned matrix include:

$$
\lambda= \pm \frac{1}{2}\left(I_{a}+J_{w} \pm \sqrt{\left(I_{a}+J_{w}\right)^{2}-4\left(I_{a} J_{w}-I_{w} J_{a}\right)}\right)
$$

The soliton is stable when the eigenvalues have no positive actual value. It can be indicated that the equations governing the above disturbances are as follows:

$$
\left(\begin{array}{l}
p \\
q
\end{array}\right)=\left(\begin{array}{l}
p_{0} \\
q_{0}
\end{array}\right) \exp (\sqrt{\lambda} Z)
$$

where $p_{0}=p(Z=0)$ and $q_{0}=q(Z=0)$.

The impact of disturbances on the pulse width, $a$, for different topological charges is demonstrated in Fig. 4. It is observed that the pulse width oscillates due to the energy and specific disturbances while propagating around the steady position. This oscillation has higher frequency for the higher topological charge.

As indicated in Fig. 5, the effect of disturbance on the pulse durability is similar to its impact on the pulse width for different topological charges. This similarity can be clearly found in the above figures.

\section{Conclusion}

Such stability in the pulse durability and pulse width results in the formation of temporal-spatial vortex solitons during the propagation in the fiber. Previously, the temporal-spatial solitons called optical bullets have been introduced. Here, since the intensity is zero in the center of vortex, we can see the hollow optical bullets like what indicated in Fig. 6.

\section{Acknowledgments}

The work described in this paper was fully supported by grants from the Institute for Advanced Studies of Iran. The authors would like to express genuinely and sincerely thanks and appreciated and their gratitude to Institute for Advanced Studies of Iran. Also, the authors appreciate valuable discussions given by M. Ghorbani, Institute for Advanced Studies (IAS).

\section{References}

Agrawal G. P. (1989). Nonlinear Fiber Optics. Second Edition, Academic Press, Boston. 
Bergé L. (1998). Wave collapse in physics: principles and applications to light and plasma waves. Physics Reports, 303, pp. 259-370. http://dx.doi.org/10.1016/S0370-1573(97)00092-6

Berry M. V. (1984). Quantal Phase Factors Accompanying Adiabatic Changes. Proc. R. Soc. A., London, 392, pp. 45. http://rspa.royalsocietypublishing.org/content/392/1802/45.short

Boyd R. W. (2003). Nonlinear Optics. Second Edition, Academic Press, Boston.

Butylkin V. S., Kaplan A. E., Khronopulo Y. G., \& Yakubovich E. I. (1989). Resonant Nonlinear Interaction of Light with Matter. Springer, Berlin.

Carlsson A. H., Malmberg J. N., Anderson D., Lisak M., Ostrovskaya E. A., Alexander T. J. \& Kivshar Y. S. (2000). Linear and nonlinear waveguides induced by optical vortex solitons, Optics Letters, 25, 9, pp. 660-662. http://dx.doi.org/10.1364/OL.25.000660

Chaos, Solitons \& Fractals, 4, pp. 1783-1796. http://dx.doi.org/10.1016/0960-0779(94)90111-2

Chen Y., \& Atai J. (1992). Gray and dark modes in nonlinear waveguides. J. Opt. Soc. Am. B. 9, pp. 2252-2257. http://dx.doi.org/10.1364/JOSAB.9.002252

Desyatnikov A. S. \& Kivshar Y. S. (2001). Necklace-Ring Vector Solitons. Phys. Rev. Lett., 87, pp. 033901-033904. http://link.aps.org/doi/10.1103/PhysRevLett.87.033901

Desyatnikov A. S., Kivshar Y. S., \& Torner L. (2005). Optical vortices and vortex solitons. Progress in Optics. 47, pp. 291-391. http://dx.doi.org/10.1016/S0079-6638(05)47006-7

Desyatnikov A., Maimistov A. \& Malomed B. (2000). Three-dimensional spinning solitons in dispersive media with the cubic-quintic nonlinearity. Phys. Rev. E., 61, pp. 3107-3113. http://link.aps.org/doi/10.1103/PhysRevE.61.3107

Hasegawa A., \& Tappert F. (1973). Transmission of stationary nonlinear optical pulses in dispersive dielectric fibers. I. Anomalous dispersion. Applied Physics Letters, 23, pp. 142-144. http://dx.doi.org/10.1063/1.1654836

Iannone E., Matera F., Mecozzi A., \& Settembre M. (1998). Nonlinear Optical Communication Networks. Chap.5.,Wiley, New York.

Jackiw R., \& So-Young P. (1990). Soliton solutions to the gauged nonlinear Schrödinger equation on the plane. Phys. Rev. Lett., 64, pp. 2969-2972. http://link.aps.org/doi/10.1103/PhysRevLett.64.2969

Kivshar Y. S. \& Peyrard M. (1992). Modulational instabilities in discrete lattices. Phys. Rev. A., 46, pp. 3198-3205. http://link.aps.org/doi/10.1103/PhysRevA.46.3198

Kivshar Y. S., \& Agrawal G. P. (2003). Optical Solitons: From Fibers to Photonic Crystals. Chap. 4 and 8, Academic, San Diego.

Kivshar Y. S., \& Yang X. (1994). Dark solitons on backgrounds of finite extent. Optics Communications, 107, pp. 93-98. http://dx.doi.org/10.1016/0030-4018(94)90109-0

Kivshar Y. S., \& Yang X. (1994). Ring dark solitons. Phys. Rev. E., 50, pp. R40-R43. http://link.aps.org/doi/10.1103/PhysRevE.50.R40

Kivshar Y. S., Christou J., Tikhonenko V., Luther-Davies B., \& Pismen L. M. (1998). Dynamics of optical vortex solitons. Optics Communications, 152, pp.198-206. http://dx.doi.org/10.1016/S0030-4018(98)00149-7

Kivshar Y.S. \& Luther-Davies B. (1998). Dark optical solitons: physics and applications. Physics Reports, 298, pp. 81-197. http://dx.doi.org/10.1016/S0370-1573(97)00073-2

Kosterlitz J. M., \& Thouless D. J. (1973). Ordering, metastability and phase transitions in two-dimensional systems. J. Phys. C., 6, pp. 1181 -1203. http://dx.doi.org/10.1088/0022-3719/6/7/010

Królikowski W., Luther-Davies B., Denz C., \& Tschudi T. (1998). Annihilation of photorefractive solitons. Opt. Lett., 23, pp. 97-99. http://dx.doi.org/10.1364/OL.23.000097

Kuznetsov E. A., \& Turitsyn S. K. (1988). Soliton instability and collapse in media with defocusing nonlinearity. Zhurnal Eksperimental'noi $i$ Teoreticheskoi Fiziki, 94, pp. 119-129. http://adsabs.harvard.edu/abs/1988ZhETF..94..119K

Kuznetsov E. A., Rubenchik A. M., \& Zakharov V. E. (1986). Soliton stability in plasmas and hydrodynamics. Physics Reports, 142, pp. 103-165. http://dx.doi.org/10.1016/0370-1573(86)90016-5 
Law C. T. \& Swartzlander Jr. G. A. (1994). Polarized optical vortex solitons: Instabilities and dynamics in Kerr nonlinear media. Chaos, Solitons \& Fractals, 4, pp. 1759-1766. http://dx.doi.org/10.1016/0960-0779(94)90109-0

Lugomer S. (1998). Observation of solitons on vortex filament bush. Phys. Lett. A., 242, pp. 319-325. http://dx.doi.org/10.1016/S0375-9601(98)00175-3

Luther-Davies B., Christou J., Tikhonenko V. \& Kivshar Y. S. J. (1997). Optical vortex solitons: experiment versus theory. Opt. Soc. Am. B., 14, pp. 3045-3053. http://dx.doi.org/10.1364/JOSAB.14.003045

Luther-Davies B., Powles R., \& Tikhonenko V. (1994). Nonlinear rotation of three-dimensional dark spatial solitons in a Gaussian laser beam. Opt. Lett., 19, pp. 1816-1818. http://dx.doi.org/10.1364/OL.19.001816

Mamaev A. V., Saffman M. \& Zozulya A. A. (1996). Propagation of Dark Stripe Beams in Nonlinear Media: Snake Instability and Creation of Optical Vortices. Phys. Rev. Lett. 76, pp. 2262-2265. http://link.aps.org/doi/10.1103/PhysRevLett.76.2262

Mamaev A. V., Saffman M. \& Zozulya A. A. (1996). Propagation of light beams in anisotropic nonlinear media: From symmetry breaking to spatial turbulence. Phys. Rev. Lett., 54, pp. 870-879. http://hexagon.physics.wisc.edu/papers\%20and\%20pubs/papers\%20in\%20pdf\%5C1996\%5Cpra_filament.pdf

Mihalache D., Mazilu D., Crasovan L.-C., Towers I., Buryak A. V., Malomed B. A., Torner L., Torres J. P., \& Lederer F. (2002). Stable Spinning Optical Solitons in Three Dimensions. Phys. Rev. Lett., 88, pp. 073902-073905. http://link.aps.org/doi/10.1103/PhysRevLett.88.073902

Mihalache D., Mazilu D., Lederer F., Crasovan L.-C., Kartashov Y. V., Torner L., \& Malomed B. A. (2006). Phys. Rev. E., 74, pp. 066614-066619. http://link.aps.org/doi/10.1103/PhysRevE.74.066614

Mollenauer L. F., Stolen R. H., \& Gordon J. P. (1980). Experimental observation of Picosecond Pulse Narrowing and Solitons in Optical Fibers. Phys. Rev. Lett., 45, pp. 1095-1098. http://link.aps.org/doi/10.1103/PhysRevLett.45.1095

Neshev D. N., Alexander T. J., Ostrovskaya E. A., Kivshar Y. S., Martin H., Makasyuk I. \& Chen Z. (2004). Observation of Discrete Vortex Solitons in Optically Induced Photonic Lattices. Phys. Rev. Lett., 92, pp. 123903-123906. http://link.aps.org/doi/10.1103/PhysRevLett.92.123903

Nore C., Brachet M. E., \& Fauve S. (1993). Numerical study of hydrodynamics using the nonlinear Schrödinger equation. Physica D., 65, pp. 154-162. http://dx.doi.org/10.1016/0167-2789(93)90011-O

Novikov S., Manakov S. V., Pitaevskii L. P., \& Zakharov V. E. (1984). Theory of solitons: The Inverse Scattering Method. Consultants Bureau, New York.

Nye J. F., \& Berry M. V. (1974). Dislocation in wave trains. Proc.R. Soc. Land. A., 336, pp. 165-190. http://rspa.royalsocietypublishing.org/content/336/1605/165.short

Pismen L. M. (1999). Vortices in nonlinear fields: From liquid crystals to superfluids, from non-equilibrium patterns to cosmic strings. Clarendon Press, Oxford and New York.

Raghavan S., \& Agrawal G. P. (2000). Spatiotemporal solitons in inhomogeneous nonlinear media. Optics Communications, 180, pp. 377-382. http://dx.doi.org/10.1016/S0030-4018(00)00727-6

Rubinstein B. Y., \& Pismen L. M. (1994). Vortex motion in the spatially inhomogenous conservative Ginzburg-Landau model. Physica D., 78, pp. 1-10. http://dx.doi.org/10.1016/0167-2789(94)00119-7

Russell J. S. (1844). Report on waves, Report of the $14^{\text {th }}$ meeting of the British Association for the advanced of science. York, pp. 311-390.

Schiesser W. E. (1994). Method of lines solution of the Korteweg-de vries equation. Computers and Mathematics with Applications, 28, pp. 147-154. http://dx.doi.org/10.1016/0898-1221(94)00190-1

Shen Y. R. (1984). The Principle of Nonlinear Optics. Wiley-Interscience, New York.

Soskin M. S., \& Vasnetsov M. V. (2001). Chapter 4 Singular optics. Progress in Optics. 42, pp. 219-276. http://dx.doi.org/10.1016/S0079-6638(01)80018-4

Staliunas K. (1994). Vortices and dark solitons in the two-dimensional nonlinear Schrödinger equation

Swartzlander, Jr. G. A., \& Law C. T. (1992). Optical vortex solitons observed in Kerr nonlinear media. Phys. Rev. Lett., 69, pp. 2503-2506. http://link.aps.org/doi/10.1103/PhysRevLett.69.2503 
Tikhonenko V., Kivshar Y. S., Steblina V. V., \& Zozulya A. A. (1998). Vortex solitons in a saturable optical medium. J. Opt. Soc. Am. B. 15, pp. 79-86. http://dx.doi.org/10.1364/JOSAB.15.000079

Tilkhonenko V., Christou J., Luther-Davies B. \& Kivshar Y. S. (1996). Observation of vortex solitons creadted by the instability of dark soliton stripes. Opt. Lett., 21, pp. 1129-1131. http://dx.doi.org/10.1364/OL.21.001129

Velchev I., Dreischuh A., Neshev D., \& Dinev S. (1997). Multiple-charged optical vortex solitons in bulk Kerr media. Optics Communications, 140, pp.77-82. http://dx.doi.org/10.1016/S0030-4018(97)00172-7

Wadati M. (2001). Introduction To Solitons. Jornal of Physics and Astronomy, 57, pp. 841-847. http://dx.doi.org/10.1007/s12043-001-0002-3

Whewell W. (1833). Essay towards a first approximation to a map of cotidal lines. Phi1. Trans. R. Soc., 123, pp. $148-236$.

Winfree A. T. (1995). Persistent tangles of vortex rings in excitable media. Physica D., 84, pp. 126-147. http://dx.doi.org/10.1016/0167-2789(95)00025-Y

Young T. (1802). On the Theory of light and colours (The Bakerian lecture). Phil. Trans. R. Soc., 20, pp. 12-48.
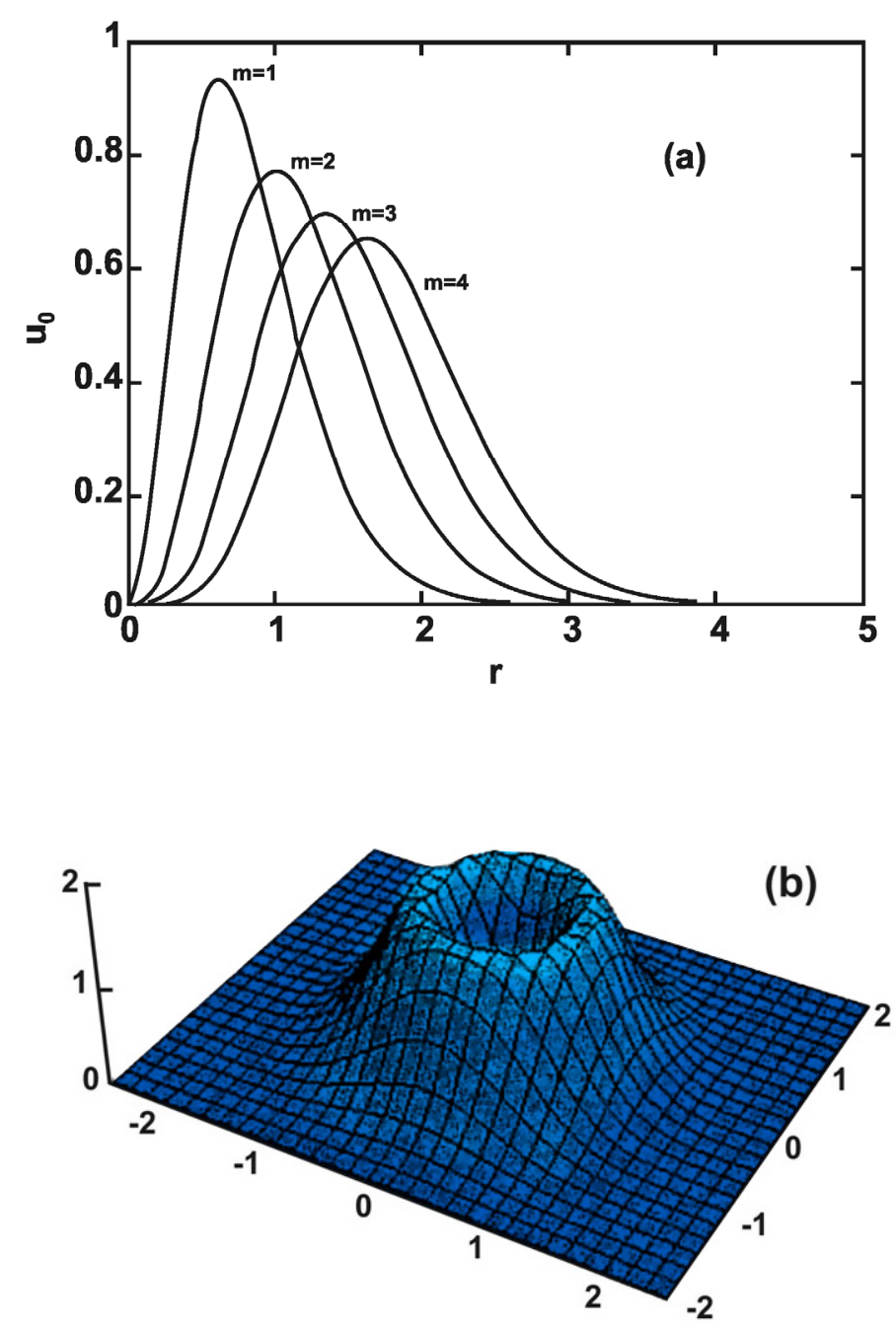

Figure 1. (a) The radial profile of the vortex solitons for different topological charges $m$; (b) the intensity distribution of vortex soliton for each topological charge 


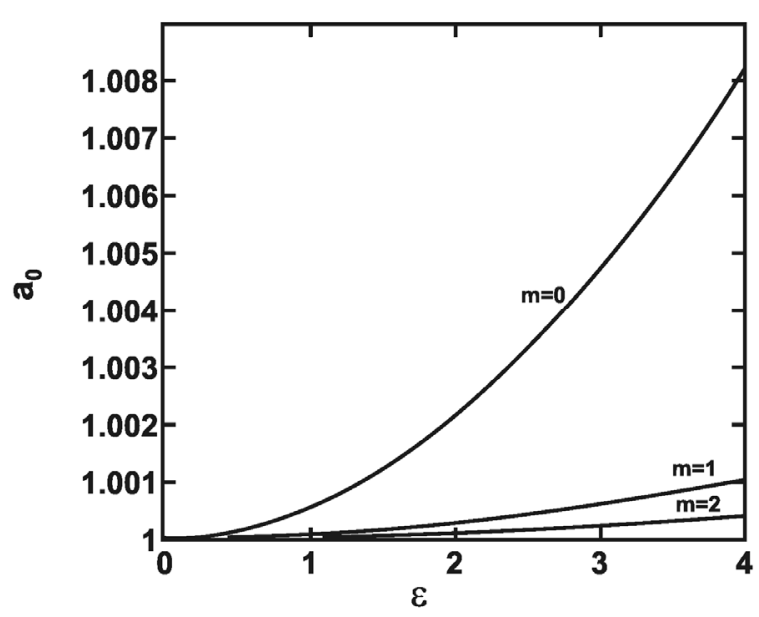

Figure 2. The changes in the spatial width according to the energy for different topological charges $m$

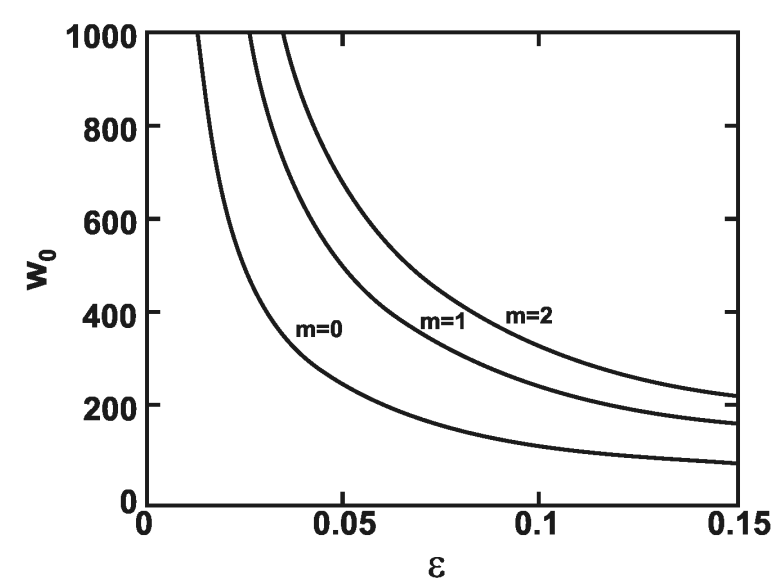

Figure 3. The changes in the pulse durability according to the energy for different topological charges $m$

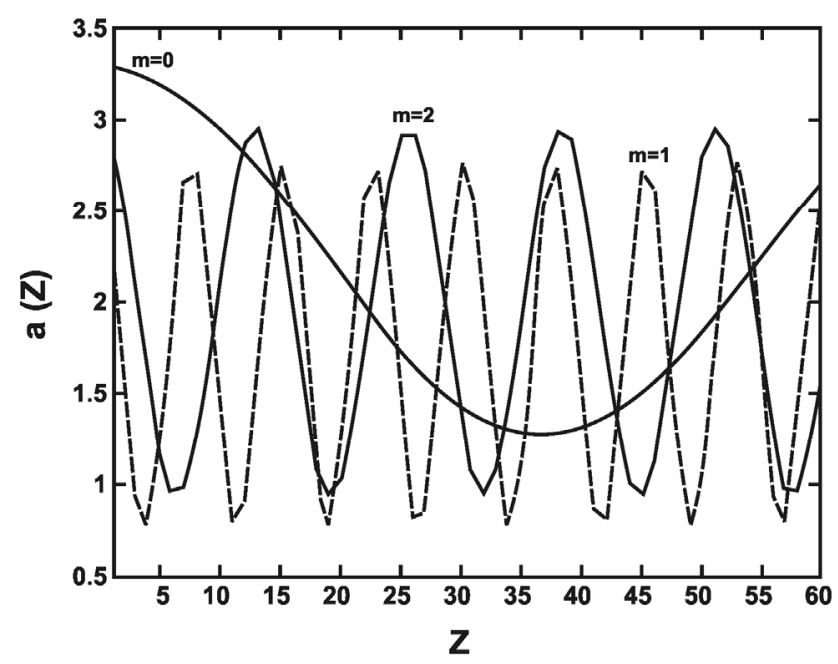

Figure 4. The effect of disturbance on the propagated pulse width in the direction $Z$ for different $m$ 


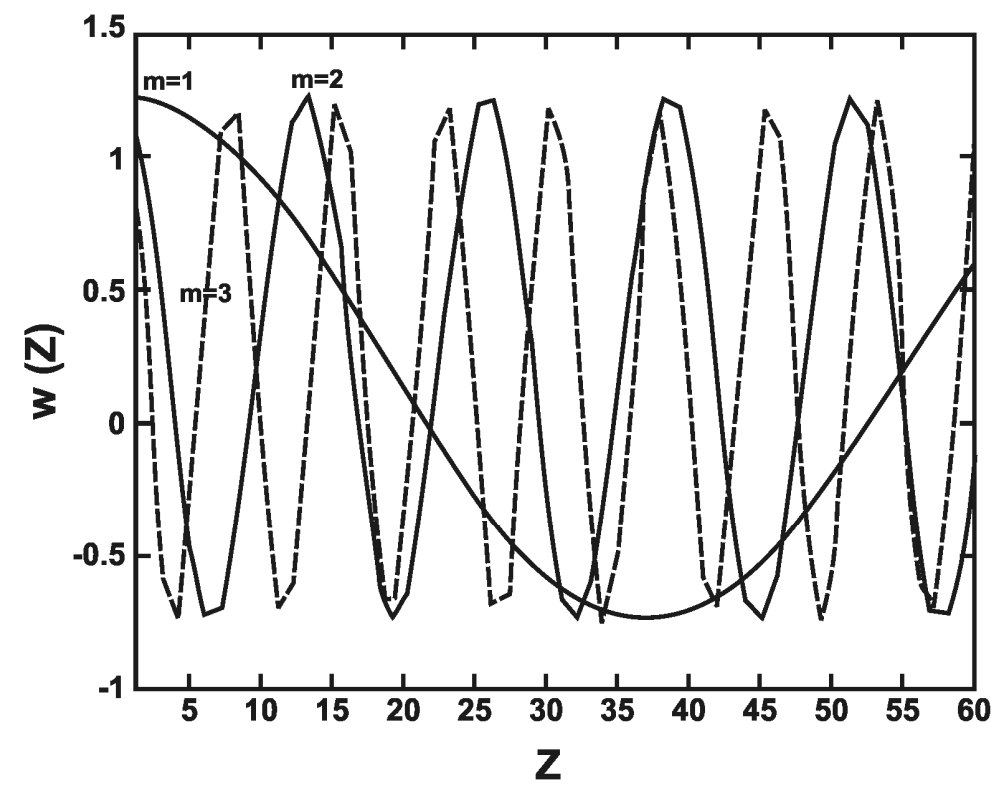

Figure 5. The effect of disturbance on the propagated pulse durability in the direction $Z$ for different $m$

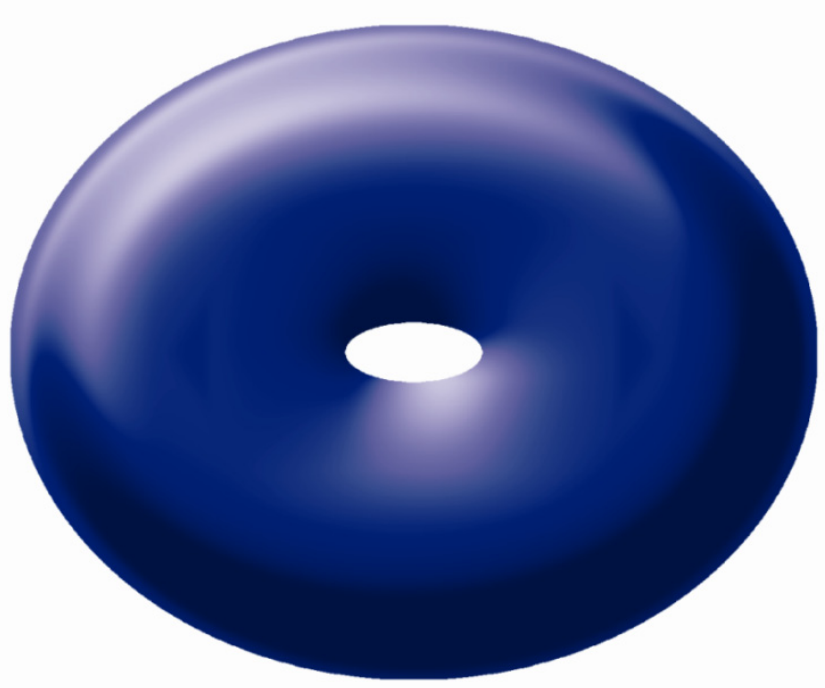

Figure 6. The vortex optical bullet 